\title{
Neuroserpin restores autophagy and promotes functional recovery after acute spinal cord injury in rats
}

\author{
ZHENG LI $^{1 *}$, FUBING LIU ${ }^{1,2^{*}}$, LIANG ZHANG ${ }^{1 *}$, YUANWU CAO $^{1}$, \\ YUNCHAO SHAO $^{1}$, XIAOFENG WANG ${ }^{1}$, XIAOXING JIANG ${ }^{1}$ and ZIXIAN CHEN ${ }^{1}$ \\ ${ }^{1}$ Department of Orthopaedics, Zhongshan Hospital, Fudan University, Shanghai 200032; ${ }^{2}$ Department of Spine Surgery, \\ The Second Xiangya Hospital, Central South University, Changsha, Hunan 410011, P.R. China
}

Received October 23, 2016; Accepted April 6, 2017

DOI: $10.3892 / \mathrm{mmr} .2017 .8249$

\begin{abstract}
This study is to reveal the characteristics of autophagy and the effect of neuroserpin (NSP) treatment on autophagy during the process of functional recovery following spinal cord injury (SCI). After the clip compress rat model of SCI had been made, autophagy-associated proteins, including LC3-II, beclin-1 and p62, were evaluated at 2, 4, 24, 72 h, and $168 \mathrm{~h}$ in the experimental group, and the sham group as control. Transmission electron microscopy (TEM) was further used for autophagy detection at 4 and $72 \mathrm{~h}$. All the male rats were randomly divided into three groups: Sham, vehicle and NSP group. NSP or an equal volume of saline vehicle was administered via intrathecal injection immediately after SCI. Each group was further divided into subgroups for the following experiments: i)Western blot (LC3-II and p62); ii) Immunofluorescent double staining (LC3/MAP-2/DAPI); iii) Nissl staining and Basso Beattie Bresnahan (BBB score) for NSP neuroprotection evaluation. Our results revealed both LC3-II and p62 expression trended upward at 24, 72 and $168 \mathrm{~h}$ after SCI. The LC3-II peaked at $72 \mathrm{~h}$, while p62 peaked at 24 h. Beclin-1 dropped significantly at 72 and $168 \mathrm{~h}$. TEM results showed that autophagosomes largely accumulated at $72 \mathrm{~h}$ after SCI when compared with the sham group. Western blot analysis showed that LC3-II and p62 were markedly decreased with NSP treatment at $72 \mathrm{~h}$ after injury compared with that of the vehicle-group. Immunofluorescent double labeling indicated that accumulation of autophagosomes was
\end{abstract}

Correspondence to: Dr Zixian Chen or Dr Xiaoxing Jiang, Department of Orthopaedics, Zhongshan Hospital, Fudan University, 180 Fenglin Road, Shanghai 200032, P.R. China

E-mail: chen.zixian@zs-hospital.sh.cn

E-mail: jiang.xiaoxing@zs-hospital.sh.cn

*Contributed equally

Abbreviations: NSP, neuroserpin; SCI, spinal cord injury; TEM, transmission electron microscopy; SD, Sprague-Dawley

Key words: autophagy, spinal cord injury, neuroserpin, autophagic flux, LC3, P62, beclin-1 reduced in the NSP group. Further, post-SCI treatment with NSP improved the BBB scale and increased the number of anterior horn motor neurons. Together, this study demonstrates that autophagic flux is impaired, meanwhile NSP restores autophagic flux and promotes functional recovery after SCI in rats.

\section{Introduction}

Spinal cord injury (SCI) leading to either temporary or permanent disability always undergoes two pathological phases: primary injury and secondary injury (1). Primary injury is always instantaneous and irreversible. Secondary injury involves multiple pathological processes such as edema, initiation of an ischemic cascade, inflammation and apoptotic cell death, and can occur over minutes or weeks following the injury. Currently, the study and treatment of SCI are mainly focused on reducing secondary injury and promoting neuronal regeneration (1-4).

Autophagy is a highly conserved process that can be induced by a variety of conditions such as hypoxia, hunger and mechanical damage (5). Previous studies have shown that autophagy plays an important role in SCI and is closely associated with apoptosis. Autophagy can reduce neuronal damage and promote locomotor recovery by inhibiting apoptosis following SCI in rats. Further, disruption of autophagy may contribute to neuronal cell death (6-8). However, the role of autophagy in SCI is still controversial and may vary depending on the type or degree of SCI $(7,9,10)$.

Neuroserpin (NSP) is a member of the serine proteinase inhibitor (serpin) family and is widely expressed in the nervous system, in areas such as the cerebral cortex, hippocampus and the spinal cord $(11,12)$. NSP is a tissue-type plasminogen activator (t-PA) inhibitor and has been shown to reduce cerebral infarct volume and protect neurons from ischemia-induced apoptosis by preventing the harmful effects of t-PA activation. However, NSP can also protect neurons from death independently of t-PA inhibition, though the molecular mechanisms underlying this effect are still poorly understood $(13,14)$. In the present study, we aimed to build a clip compress model of SCI and identify the characteristic markers of autophagy following the injury as well as the effect of NSP treatment on autophagy. We hope to provide a 
novel therapeutic strategy for the treatment of SCI based on intervention of autophagy.

\section{Materials and methods}

Animals. Adult male Sprague-Dawley (SD) rats weighing 250-300 g were provided by the Department of Laboratory Animal Science of Fudan University. All animal interventions strictly complied with the regulations of the Animals Ethics Committee of Fudan University. Animals were kept in a specific pathogen-free (SPF) barrier system for 1 week to adapt to the environment and were fed ad libitum. The room temperature was held constant at $27^{\circ} \mathrm{C}$.

SCI model. Before the operation, animals were anesthetized with $10 \%$ chloral hydrate via intraperitoneal injection $(0.3 \mathrm{ml} / 100 \mathrm{~g})$. After sterilization with iodophor, they were placed in a prone position. T10 was located according to the bony process on the dorsum, and the skin and muscle were cut and separated to expose the lamina. A laminectomy was performed with a mini-toothed clamp to fully expose the spinal cord at T9-T10, then a smooth vascular clamp (30 g force; Oscar, Shanghai, China) was used to compress the spinal cord vertically for $1 \mathrm{~min}$ (15-17). Postoperative care included manual assisted urination twice daily. If hematuresis was noted, penicillin was administered to prevent infection. The rat hairs were kept dry, and food and water were placed so that the animals had easy and free access.

NSP treatment.Immediately following the injury, rats randomly received NSP (20 $\mu \mathrm{g} / \mathrm{ml}$, intrathecal injection; PeproTech) $25 \mu \mathrm{l}$ or an equal volume of saline vehicle. A group of sham-operated rats was included as an additional control group.

Western blot analysis. At different time-points (2, 4, 24, $72 \mathrm{~h}$ and 7 days) after SCI and $3 \mathrm{~d}$ after the sham operation $(n=4)$, rats were anesthetized with $10 \%$ chloral hydrate, and an intracardiac perfusion was performed with normal saline. A spinal cord segment $(0.5 \mathrm{~cm})$ across the epicenter of injury was collected and rapidly stored at $-80^{\circ} \mathrm{C}$ for further study. Protein was extracted with RIPA [150 mM NaCl, $50 \mathrm{mM}$ Tris (8.0), 1\% NP40, 1\% sodium deoxycholate, 0.1\% SDS pH 7.4] that contained a protease inhibitor cocktail (Roche, Germany). The protein concentration was determined with a BCA protein assay kit (Tiangen, China). A total of $40 \mu \mathrm{g}$ of protein was separated via $12.5 \%$ polyacrylamide gel (Promoton, Shanghai, China) and transferred onto a PVDF membrane. The membrane was blocked with $5 \%$ skim milk in TBST (TBS contain $0.1 \%$ Tween-20) for $1 \mathrm{~h}$, then incubated with primary antibody rabbit anti-LC3 (1:3,000; Sigma-Aldrich, St. Louis, MO, USA), mouse anti-p62 (1:2,000; Abcam, Cambridge, UK), rabbit anti-beclin1 (1:1,000; Novus Biologicals, Littleton, $\mathrm{CO}$, USA) at $4^{\circ} \mathrm{C}$ overnight. Thereafter, the membrane was incubated with secondary antibody linked to horseradish peroxidase (HRP) (goat anti-mouse or goat anti-rabbit, 1:5,000; Sigma-Aldrich) for $2 \mathrm{~h}$ at room temperature. $\beta$-actin (mouse anti- $\beta$-actin, 1:5,000; Sigma-Aldrich) was set as an internal reference. Immunoreactive bands were detected with an enhanced chemiluminescence system (ECL kit; Sangon Biotech Co., Ltd., Shanghai, China). The image was analyzed with Quantity One software (version 4.62; Bio-Rad, Hercules, CA, USA). A commercial molecular weight marker ranging from 10 to $250 \mathrm{kDa}$ was used to locate the target protein.

Transmission electron microscopy. To evaluate the ultrastructural changes following SCI, transmission electron microscopy (TEM) was further used for autophagy detection at $4 \mathrm{~h}$ and 3 days and compared with the sham group $(n=3$ for each group). After anesthesia, the rats were perfused with normal saline $\left(37^{\circ} \mathrm{C}\right)$ to clean the blood. Then, $50 \mathrm{ml}$ of fixed liquid (0.1 M phosphate buffer ( $\mathrm{pH}$ 7.4) containing $4 \%$ paraformaldehyde and $1 \%$ glutaraldehyde, $37^{\circ} \mathrm{C}$ ) was perfused quickly, after which 250 to $300 \mathrm{ml}$ of fixed liquid $\left(4^{\circ} \mathrm{C}\right)$ was perfused slowly for 10 to 15 mins. The targeted spinal cord ( $2.5 \mathrm{~mm}$ around the epicenter) was then quickly removed, and the anterior horn of the spinal cord was cut into $1 \mathrm{~mm}^{3}$ pieces and postfixed in $2.5 \%$ glutaraldehyde for more than $3 \mathrm{~h}$ at $4^{\circ} \mathrm{C}$. Subsequently, according to the standard procedures, the pieces were osmicated with $1 \%$ osmium tetroxide for $1 \mathrm{~h}$, dehydrated in an ethanol series and embedded in epon. Ultrathin sections were cut and stained with uranyl acetate and lead citrate in that order. An electron microscope (Hitachi, Tokyo, Japan) was used to evaluate the ultrastructural changes.

Immunofluorescence double staining. At 3 days following $\mathrm{SCI}$, tissue sections from the sham-group, the vehicle-group and the NSP-group were collected. After anesthesia, the rats (SCI and Sham group, $n=3$ ) were placed in the supine position. An intracardiac perfusion was performed with $0.01 \mathrm{M}$ phosphate-buffered saline (PBS, pH 7.4) and followed with 4\% paraformaldehyde (PFA in 0.1 M PBS, pH 7.4). A spinal cord segment ( $2 \mathrm{~cm}$ length) across the epicenter of the injury was harvested and fixed with $4 \%$ PFA overnight. The tissue was subsequently soaked in $30 \%$ sucrose solution at $4^{\circ} \mathrm{C}$ until it sank to the bottom. Then, the spinal cord was embedded in OCT (Polysciences, Inc., Warrington, PA, USA) at $-20^{\circ} \mathrm{C}$ and cut into a series of $10 \mu \mathrm{m}$ transverse sections. Ten sequential sections at $50 \mu \mathrm{m}$ intervals were collected, which spanned a $500 \mu \mathrm{m}$ length across the epicenter site. Frozen sections were placed on poly-L-lysine coated slides and permeabilized in $0.1 \mathrm{~mol} / \mathrm{l} \mathrm{PBS}$ ( $\mathrm{pH} 7.40$ ) containing $0.25 \%$ Triton $\mathrm{X}-100$ for $30 \mathrm{~min}$, blocked with $3 \% \mathrm{BSA}$ in $0.1 \mathrm{~mol} / 1 \mathrm{PBS}$ containing $0.05 \%$ Tween for $30 \mathrm{~min}$ at room temperature, and then incubated with rabbit anti-LC3 antibody (1:200; Sigma-Aldrich) and mouse anti-MAP2 (1:200; ProteinTech, Chicago, IL, USA) overnight at $4^{\circ} \mathrm{C}$. After washing in PBS, the sections were incubated in a mixture of goat anti-rabbit TRITC and goat anti-mouse FITC fluorescence-conjugated secondary antibodies (1:500; Invitrogen, Carlsbad, CA, USA) for $2 \mathrm{~h}$ at room temperature. The anterior horn in these slides was observed under a laser confocal microscope (Nikon, Tokyo, Japan).

Nissl staining. At 14 days, tissue from the sham-, the vehicle- and the NSP-groups were cut into a series of $50 \mu \mathrm{m}$ transverse sections. Ten sequential sections at $50 \mu \mathrm{m}$ intervals were collected, which spanned a 1,000 $\mu \mathrm{m}$ length across the epicenter site. Sections were stained with Nissl staining solution (Beyotime, Nanjing, China) according to the manufacturer's instructions. In brief, the spinal cord was stained with Nissl solution for $10 \mathrm{~min}$, followed by two 5 min washes with $95 \%$ 

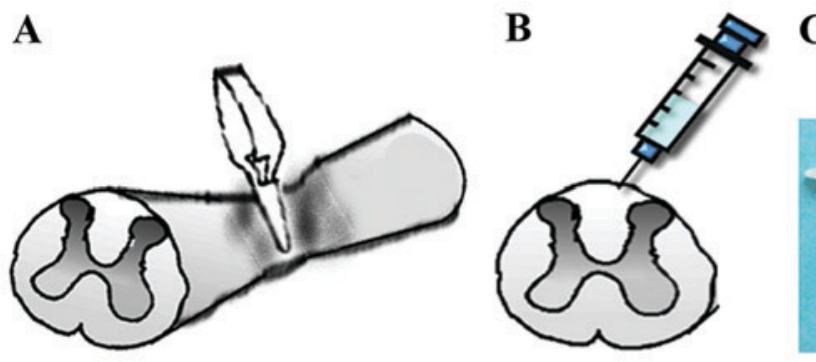

C

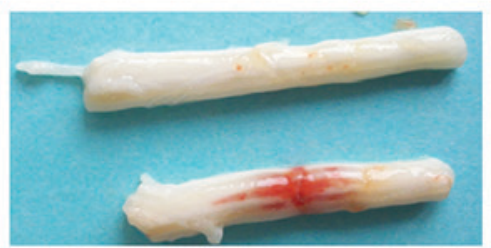

D

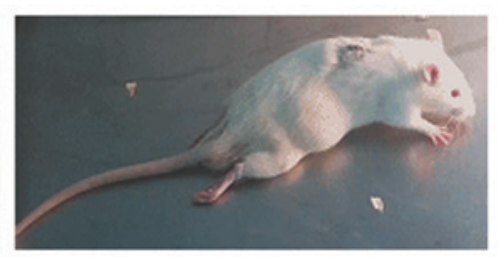

Figure 1. Expression of LC3-II and p62 in SCI rats. (A) Smooth vascular clamp was used to compress the spinal cord vertically for 1 min. (B) Intrathecal injection was shown: $25 \mu \mathrm{l}$ neuroserpin (NSP) or an equal volume of saline vehicle was injected by microinjector. (C) A strip hemorrhagic band was visible after the clamp removed. (D) Double lower limb paralysis was observed after spinal cord injury.
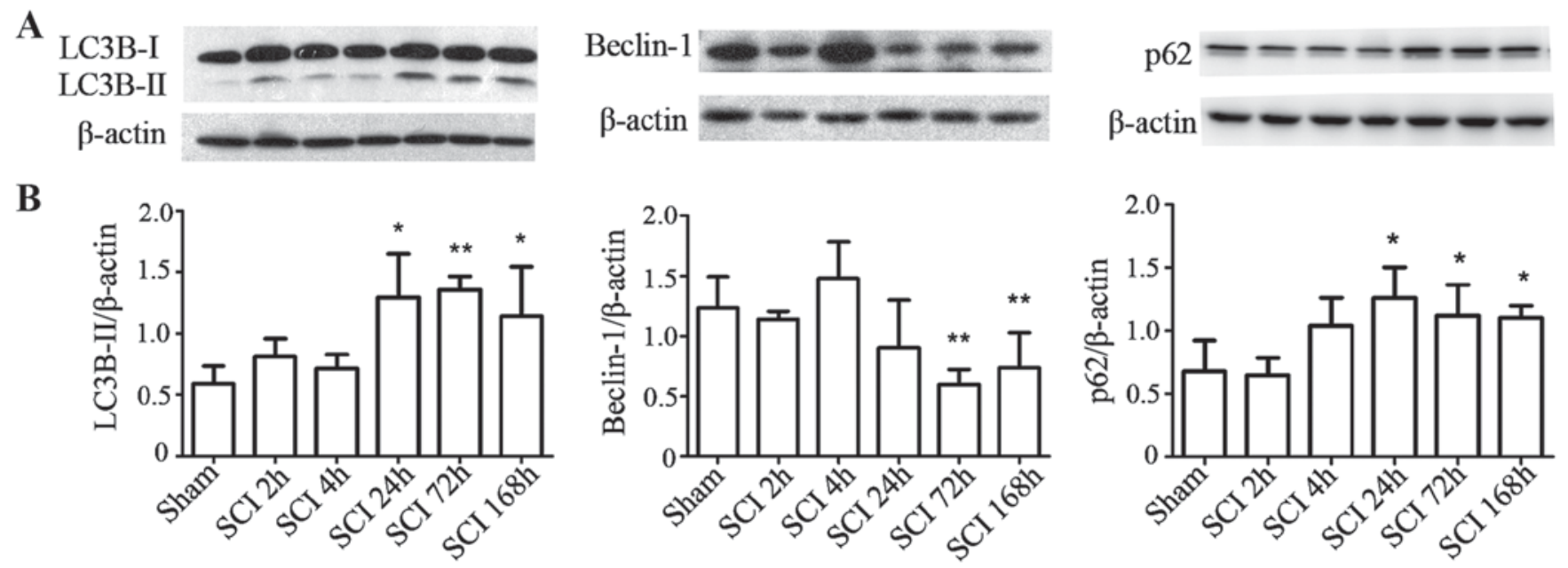

Figure 2. SCI increased LC3-II and p62 protein levels. (A) Western blot analyses of LC3-II, beclin-1 and p62 at different time-points after SCI. (B) Quantitative analysis of western blot analyses. The levels of Lc3-II and p62 were upregulated at 24, 72 and $168 \mathrm{~h}$ after SCI compared with the sham-operated group, while beclin-1 significantly dropped at 72 and $168 \mathrm{~h}$. Mean $\pm \mathrm{SD}$ ( $\mathrm{n}=4$ /group). ${ }^{*} \mathrm{P}<0.05,{ }^{* *} \mathrm{P}<0.01$ vs. sham control.

alcohol. The sections were subsequently made diaphanous twice with dimethylbenzene for $5 \mathrm{~min}$. The overall structure from each group was compared. The number of anterior horn motor neurons were also counted and compared.

Behavioral analysis. Basso-beattle-bresnahan (BBB) locomotor rating scale was applied to evaluate motor function recovery. The scale ranged from 0 to 21 . A score of 0 indicates complete paralysis of the hind limb, while a score of 21 indicates normal locomotion. The rats were set in an open-field, and two experienced researchers who were blind to the operation participated in the evaluation $(24,72,168 \mathrm{~h}$ and 14 days, for 5 min during each period of observation). The NSP-group and vehicle-group scores were compared.

Statistical analysis. Data is presented as the mean \pm SD and compared using one-way analysis of variance (ANOVA). All experimental data were analyzed using SPSS 20.0 (SPSS, Inc., Chicago, IL, USA). $\mathrm{P}<0.05$ was considered statistically significant.

\section{Results}

SCI model. The animals were anesthetized with $10 \%$ chloral hydrate via intraperitoneal injection $(0.3 \mathrm{ml} / 100 \mathrm{~g})$. A smooth vascular clamp (30 $\mathrm{g}$ force; Oscar) was used to compress the spinal cord vertically for $1 \mathrm{~min}$ (Fig. 1A). The sham group underwent the same surgical procedure, but no clip compression was applied to the spinal cord. After adequate hemostasis, the incision was closed in layers. A strip hemorrhagic focus was visible after the clamp was removed (Fig. 1C). When the animals awakened, they were evaluated for double lower limb paralysis and the presence of any obstacles to urination (Fig. 1D).

Expression of LC3-II and p62 in SCI rats. To investigate the changes in autophagy at different time-points following SCI, LC3-II and p62 protein levels were measured by western blot analysis. Compared with the sham-operated group, LC3-II and p62 protein levels increased at 1, 3, and 7 days after injury in the experimental group $(\mathrm{P}<0.05)$, while Beclin-1 significantly dropped at 3 and 7 days post-surgery in the SCI compared with the sham group $(\mathrm{P}<0.01$; Fig. $2 \mathrm{~A}$ and $\mathrm{B})$.

Ultrastructural examination for autophagy in SCI rats. Normal mitochondria, endoplasmic reticulum and regular myelinated axons were observed in TEM from the sham-group rats in the areas corresponding to those in the SCI group, but rarely in autophagic vacuoles (Fig. 3A and C). In contrast, disordered structure of mitochondria, myelinated axons, and 

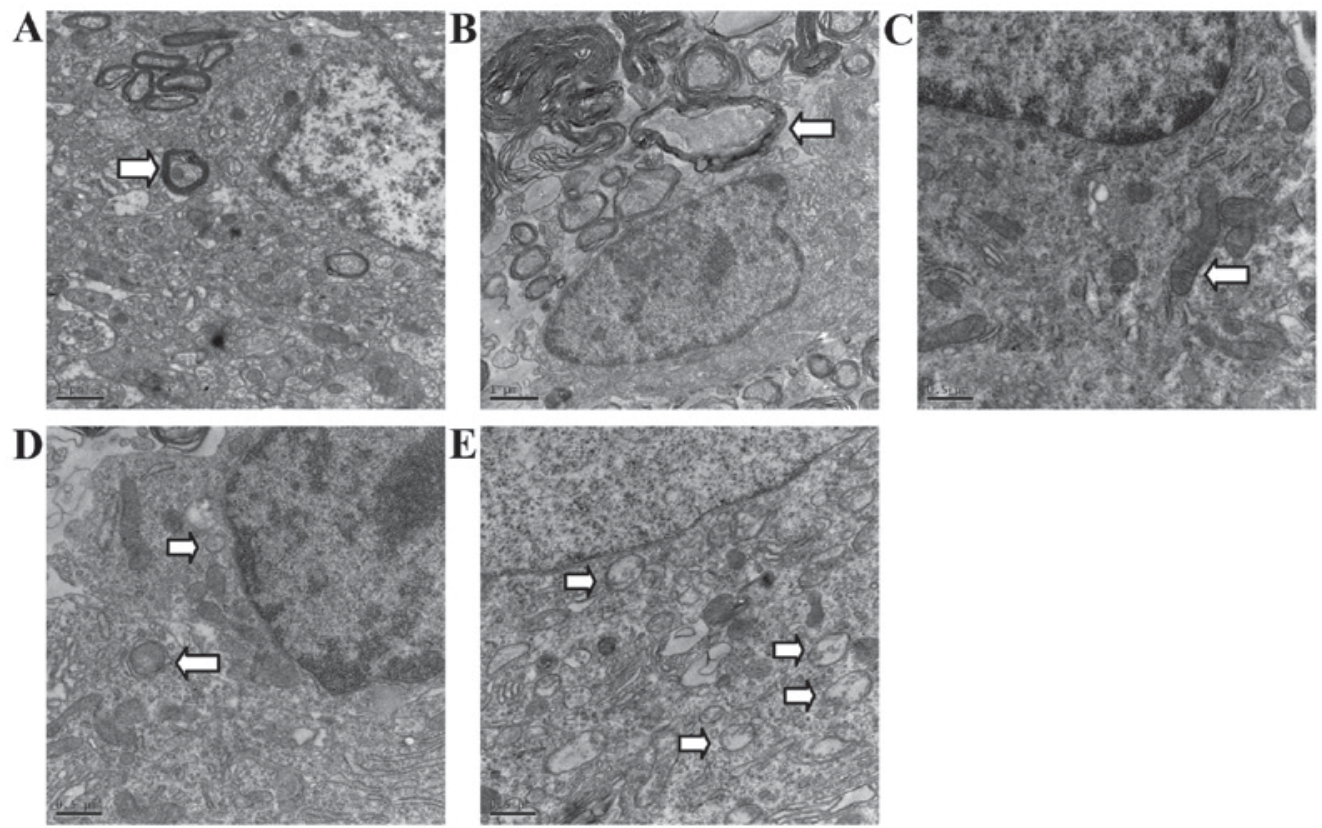

Figure 3. Ultrastructural demonstration of autophagy after SCI in rats. Electron micrographs of the anterior horn area near the epicenter in sham-group (A and C) and SCI-group (B, D and E). (A and C) Show regular, lamellar distribution of myelin (arrowhead) and normal neurons in the sham-group containing intact mitochondria (arrowhead), but few cytoplasmic vacuoles. (B) Shows the disorder of myelin (arrowhead) and structure at $72 \mathrm{~h}$ after SCI, numerous neural structures were lost or destroyed. (D and E) Demonstrate the double-membrane structure autophagosome (arrowhead) formed and accumulated at 2 and $72 \mathrm{~h}$, respectively. Autophagosomes were largely accumulated at $72 \mathrm{~h}$ after SCI. Scale bars, $1 \mu \mathrm{m}$ (A and C); $500 \mathrm{~nm}$ (B, D and E).

numerous autophagic vacuoles with a double-membrane structure and parts of cytoplasmic organelles were detected near the epicenter of the rats at $2 \mathrm{~h}$, and especially $72 \mathrm{~h}$ after SCI. Autophagosomes were largely observed in the cytoplasm near the nuclei (Fig. 3B, D and E).

NSP reduced the levels of LC3-II and p62. Western blot analysis indicated that NSP treatment decreased LC3-II and p62 protein levels at $72 \mathrm{~h}$ after SCI when compared with the vehicle-treated rats $(\mathrm{P}<0.01$; Fig. $4 \mathrm{~A}$ and $\mathrm{B})$. Double immunofluorescence staining of the epicenter sections also showed that at $72 \mathrm{~h}$ after the injury, LC3 was increased in the SCI compared with the sham-group, and displayed a punctate distribution in the neurons. NSP decreased the LC3 expression as evidenced by the decreased numbers of LC3 dots under confocal microscopy (Fig. 4C).

NSP promotes neuroprotection and functional recovery following SCI. At 14 days after injury, there was an increase in the number of motor neurons near the injury epicenter in NSP treated rats compared with the vehicle group. Sections located a $2 \mathrm{~mm}$ distance from the epicenter revealed that NSP increased the number of motor neurons However, there was also a significant decrease in the number motor neurons in the NSP-treated rats compared with sham-group (Fig. 5A-E). Representative photomicrographs of motor neurons from NSP-treated and vehicle-treated rats are shown in Fig. 5A-C. The BBB score was measured at 1, 3, 7 and 14 days (Fig. 5F). From 7 to 14 days after injury, the scores in the NSP-treated rats were significantly higher than those in the vehicle-treated group. However, the scores of the NSP-treated and vehicle-treated groups both declined compared with those of the sham-group.

\section{Discussion}

Previous studies have identified that autophagy could reduce neuronal damage and promote locomotor recovery via inhibition of apoptosis after SCI in rats. Here, we showed that NSP can restore autophagic flux impairment and reduce autophagosomes accumulation by decreasing LC3-II and p62 protein levels at $72 \mathrm{~h}$ after SCI. As expected, NSP significantly reduced neuronal damage and promoted locomotor function after SCI. To the best of our knowledge, this is the first study to demonstrate a link between NSP-mediated neuroprotection and autophagy following SCI.

Autophagy has been reported to be involved in a series of neurological disease, such as Alzheimer's disease, Parkinson's disease, traumatic brain injury and spinal cord injury (18-20). The process of autophagy entails the degradation of expired proteins or damaged organelles to maintain the stability of the intracellular environment, which is important for cells to survive a temporary crisis. However, autophagy can also induce cell death, which is often referred to as type II programmed cell death (21). Autophagy is also closely associated with apoptosis. The role that autophagy plays in SCI is still controversial and varies depending on the type and extent of SCI as well as the SCI animal model being studied. A study by Chen et al showed that autophagy was activated after SCI (in a contusion rat model, $10 \mathrm{~g}$ rod dropped from $25 \mathrm{~mm}$ height) and inhibited by methylprednisolone suggesting that methylprednisolone may protect neurons by inhibiting autophagic cell death (22). However, Sekiguchi et al reported that rapamycin also promotes autophagy and reduces neural tissue damage after SCI (in a contusion mice model, $10 \mathrm{~g} \mathrm{x} 3 \mathrm{~mm}$ ) (10). Another study by Tang et al demonstrated that autophagy reduces neural damage and promotes locomotor recovery via 
A
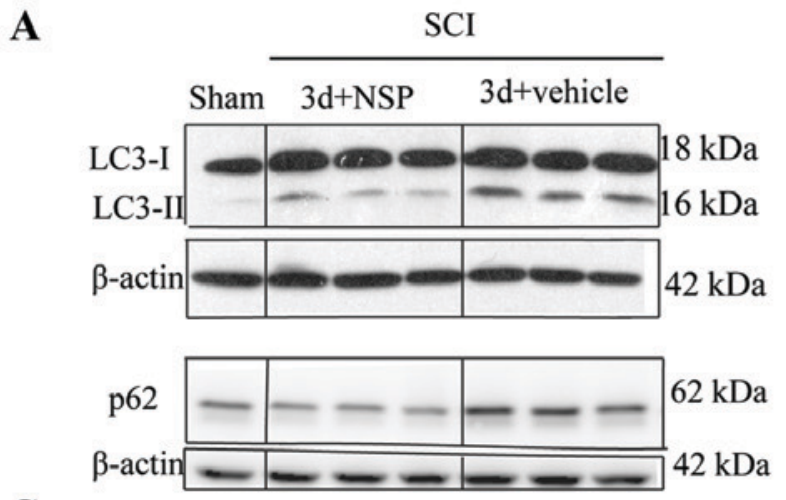

C

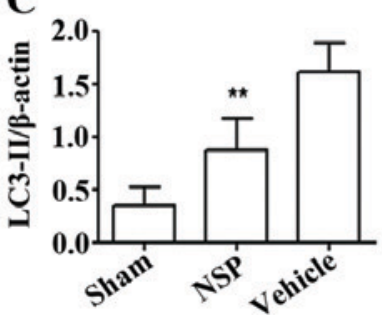

B

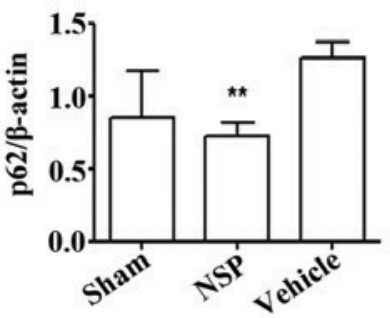

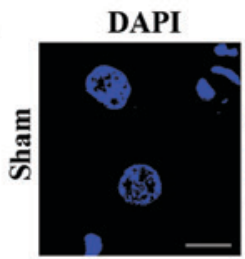
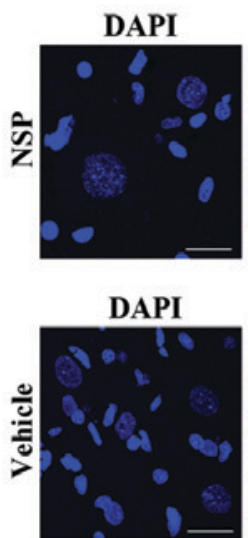

MAP-2

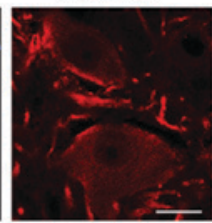

MAP-2

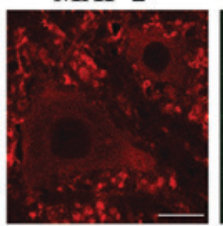

MAP-2

LC-3

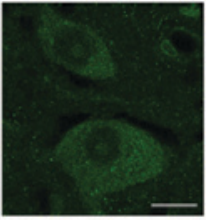

LC-3

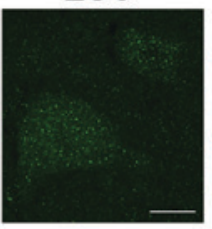

LC-3
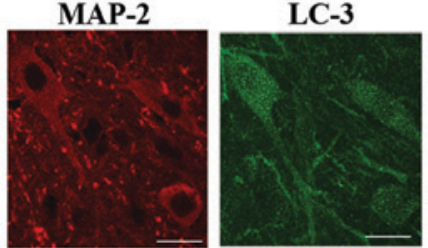

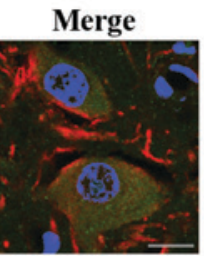

Figure 4. NSP can restore disruption of autophagic flux after SCI. (A) Western blot analyses of LC3 and p62 protein in the spinal cord at $72 \mathrm{~h}$ after SCI (B) Quantitative analysis of western blot analyses indicated that NSP reduces LC3-II and p62 protein levels at $72 \mathrm{~h}$ after SCI. Mean \pm SD (n=6/group). ${ }^{*} \mathrm{P}<0.05,{ }^{* *} \mathrm{P}<0.01$ compared with the vehicle-group. (C) Representative confocal images of LC3 and MAP-2 immunofluorescence double staining in the sham group, NSP-treated and vehicle-treated animals. Vehicle-treated rats exhibited increased dots of LC3 compared with the sham control at $72 \mathrm{~h}$ after SCI. NSP decreased the dots of LC3 vs. vehicle control (n=3/group; scale bars, $50 \mu \mathrm{m}$ ).
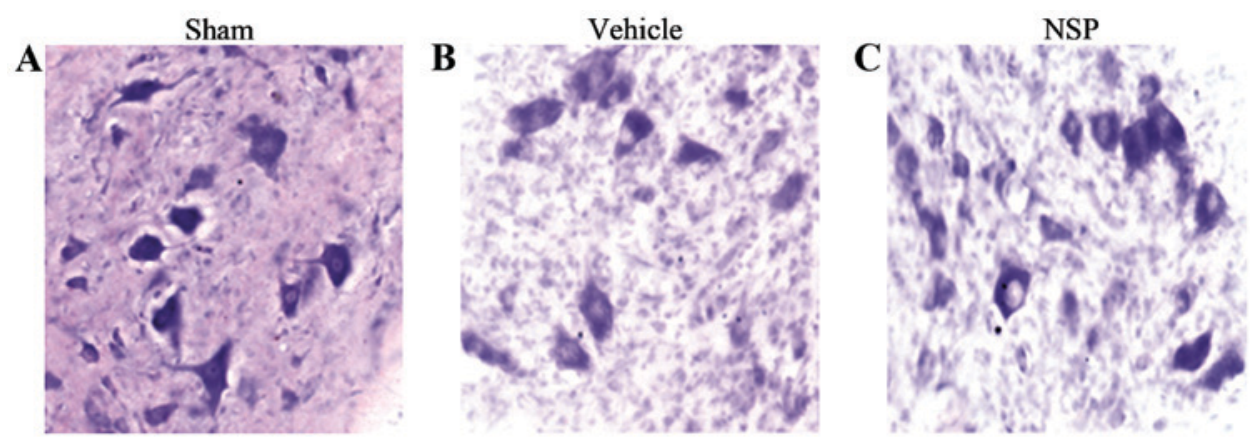

D

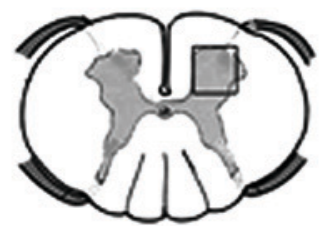

$\mathbf{E}$

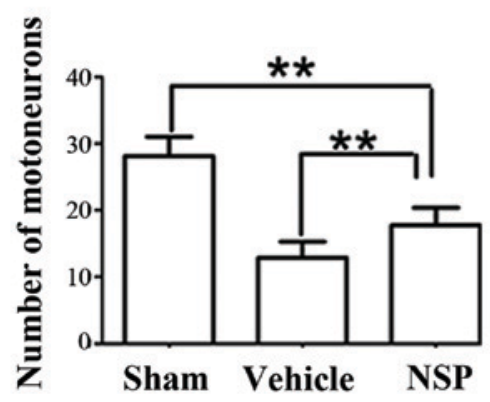

F

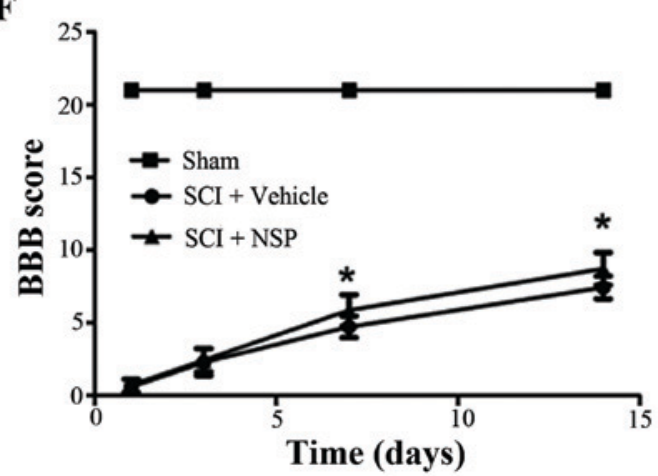

Figure 5. NSP reduced motor neuron loss after SCI. (A-C) representative microphotographs showing Nissl-stained anterior horn at 14 days after SCI. Sections located $2 \mathrm{~mm}$ distance from the epicenter revealed that NSP increased the number of motor neurons. (D) The area motor neurons counted. (E) The numbers of motor neurons in the NSP-treated animals were higher than those in the vehicle-treated animals at approximately $2 \mathrm{~mm}$ on the rostral and caudal sides of the epicenter $\left({ }^{* *} \mathrm{P}<0.01, \mathrm{n}=7 /\right.$ group; scale bars, $200 \mu \mathrm{m}$ ). (F) The BBB score was evaluated for 14 days after surgery. The scores were significantly higher in the NSP-treated group than in the vehicle-treated group from 7 to 14 days. Mean $\pm \mathrm{SD}(\mathrm{P}<0.05$ vs. vehicle treatment, $\mathrm{n}=7 /$ group $)$. 
inhibition of apoptosis after SCI (a hemisection model) (7). In our study, using another SCI rat model with a simple and economic clip compression, we demonstrated changes in the characteristic markers of autophagy after SCI and, further, illustrated these changes with TME.

LC3, a mammalian homolog of yeast Atg8, is the most important and reliable marker of autophagy used in research studies. During the formation of the autophagosome membrane, the conversion of LC3-I to LC3-II is considered to be one of the symbols of autophagic induction. Therefore, LC3-II is often used as a marker for autophagosomes. Beclin-1 did not strictly follow the same pattern as LC3-II and peaked at $4 \mathrm{~h}$. Previous studies have demonstrated that beclin-1 participates in the regulation of autophagy as well as playing a role in apoptosis that is not limited to autophagy (23). Our study demonstrated that LC3-II protein levels trended upwards from $24 \mathrm{~h}$ after SCI and peaked at $72 \mathrm{~h}$. p62 protein interacts with LC3-II and transports altered proteins to autophagosomes for degradation. The accumulation of $\mathrm{p} 62$ protein reflects the disruption of autophagic flux (24). Our study showed that $\mathrm{p} 62$ rises from $24 \mathrm{~h}$ after SCI. The downregulation of autophagy indicated that cells may be trapped in an energy crisis or have sustained injuries to organelles and can no longer control autophagic degradation. These cells may then proceed to undergo autophagic death or apoptosis. TEM, which can display the double-membrane autophagosome, is the gold standard for autophagy detection (25). In this study, TEM showed that autophagosomes largely accumulated at $72 \mathrm{~h}$ following compression-induced SCI. In the sham group, few autophagosomes were observed, while normal cell structure with layered myelin was ubiquitous. This was in contrast to the numerous vacuole-like structures and disordered myelin observed in the SCI group. The loss of neural structure after SCI may directly contribute to the functional impairment, which is difficult to reverse with simple drug intervention.

To minimize the secondary cell death after SCI, it is imperative that drug therapy be promptly instituted. NSP is a tissue-type plasminogen activator (t-PA) inhibitor. A previous study showed that in a focal cerebral ischemia/reperfusion model, intracortical injection of $30 \mu \mathrm{mol} / 1 \mathrm{NSP}$ reduced infarct volume by $64 \%$ from $161 \mathrm{~mm}^{3}$ in control animals to $58 \mathrm{~mm}^{3}$ in NSP-treated animals $72 \mathrm{~h}$ after reperfusion. NSP also decreased apoptotic cell counts in the ischemic penumbra by more than $50 \%$ (14). Our previous study also demonstrated that, in the early period of sustained spinal cord compression. NSP is upregulated and plays a neuroprotective role against neuronal apoptosis (12). NSP does not appear to freely cross the blood-brain barrier. In the present study, NSP was administered by intrathecal injection based on the results from previous studies in which. intracisternal or intracortical injections of NSP were used $(14,26)$. In addition, the doses of NSP used in this study were based on our previous protocols (27). Our results demonstrate that autophagic flux was restored by NSP, possibly by lowering p62 and LC3-II protein levels. Immunofluorescence double staining also indicated that the LC3 fluorescence dots, located in the cytoplasm were markedly decreased in neurons at the epicenter in the NSP group. These data indicate that NSP partially inhibited the accumulation of autophagosomes. Additionally, NSP improved functional recovery after injury and significantly increased the number of anterior horn motor neurons. Therefore, we suggest that NSP-induced neuroprotection and functional recovery following SCI may at least in part be associated with a restoration of SCI-induced autophagic flux impairment.

In conclusion, our results suggest that treatment with NSP following SCI restores the impaired autophagic flux and offers a new strategy for reducing secondary damage.

\section{Acknowledgements}

This study was supported by the National Natural Science Foundation of China (81301047).

\section{References}

1. Kwon BK, Tetzlaff W, Grauer JN, Beiner J and Vaccaro AR: Pathophysiology and pharmacologic treatment of acute spinal cord injury. Spine J 4: 451-464, 2004.

2. Yu WY and He DW: Current trends in spinal cord injury repair. Eur Rev Med Pharmacol Sci 19: 3340-3344, 2015.

3. Thuret S, Moon LD and Gage FH: Therapeutic interventions after spinal cord injury. Nat Rev Neurosci 7: 628-643, 2006.

4. Varma AK, Das A, Wallace G IV, Barry J, Vertegel AA, Ray SK and Banik NL: Spinal cord injury: A review of current therapy, future treatments, and basic science frontiers. Neurochem Res 38: 895-905, 2013.

5. Mizushima N: Autophagy: Process and function. Genes Dev 21: 2861-2873, 2007.

6. Wang ZY, Lin JH, Muharram A and Liu WG: Beclin-1-mediated autophagy protects spinal cord neurons against mechanical injury-induced apoptosis. Apoptosis 19: 933-945, 2014.

7. Tang P, Hou H, Zhang L, Lan X, Mao Z, Liu D, He C, Du H and Zhang L: Autophagy reduces neuronal damage and promotes locomotor recovery via inhibition of apoptosis after spinal cord injury in rats. Mol Neurobiol 49: 276-287, 2014.

8. Liu S, Sarkar C, Dinizo M, Faden AI, Koh EY, Lipinski MM and $\mathrm{Wu}$ J: Disrupted autophagy after spinal cord injury is associated with ER stress and neuronal cell death. Cell Death Dis 6: e1582, 2015.

9. Kanno H, Ozawa H, Sekiguchi A and Itoi E: Spinal cord injury induces upregulation of Beclin 1 and promotes autophagic cell death. Neurobiol Dis 33: 143-148, 2009.

10. Sekiguchi A, Kanno H, Ozawa H, Yamaya S and Itoi E: Rapamycin promotes autophagy and reduces neural tissue damage and locomotor impairment after spinal cord injury in mice. J Neurotrauma 29: 946-956, 2012.

11. Miranda E and Lomas DA: Neuroserpin: A serpin to think about. Cell Mol Life Sci 63: 709-722, 2006.

12. Wan S, Feng Z, Chen Z, Wang X, Cao Y, Shao Y and Jiang X: Neuroserpin upregulates in the early period of sustained spinal cord compression. Clin Lab 58: 891-896, 2012.

13. Wu J, Echeverry R, Guzman J and Yepes M: Neuroserpin protects neurons from ischemia-induced plasmin-mediated cell death independently of tissue-type plasminogen activator inhibition. Am J Pathol 177: 2576-2584, 2010.

14. Yepes M, Sandkvist M, Wong MK, Coleman TA, Smith E, Cohan SL and Lawrence DA: Neuroserpin reduces cerebral infarct volume and protects neurons from ischemia-induced apoptosis. Blood 96: 569-576, 2000.

15. Rivlin AS and Tator $\mathrm{CH}$ : Effect of duration of acute spinal cord compression in a new acute cord injury model in the rat. Surg Neurol 10: 38-43, 1978.

16. von Euler M, Seiger A and Sundström E: Clip compression injury in the spinal cord: A correlative study of neurological and morphological alterations. Exp Neurol 145: 502-510, 1997.

17. Zhang HY, Wang ZG, Wu FZ, Kong XX, Yang J, Lin BB, Zhu SP, Lin L, Gan CS, Fu XB, et al: Regulation of autophagy and ubiquitinated protein accumulation by bFGF promotes functional recovery and neural protection in a rat model of spinal cord injury. Mol Neurobiol 48: 452-464, 2013.

18. Nixon RA: The role of autophagy in neurodegenerative disease. Nat Med 19: 983-997, 2013. 
19. Luo CL, Li BX, Li QQ, Chen XP, Sun YX, Bao HJ, Dai DK, Shen YW, Xu HF, Ni H, et al: Autophagy is involved in traumatic brain injury-induced cell death and contributes to functional outcome deficits in mice. Neuroscience 184: 54-63, 2011.

20. Ghavami S, Shojaei S, Yeganeh B, Ande SR, Jangamreddy JR, Mehrpour M, Christoffersson J, Chaabane W, Moghadam AR, Kashani $\mathrm{HH}$, et al: Autophagy and apoptosis dysfunction in neurodegenerative disorders. Prog Neurobiol 112: 24-49, 2014

21. Smith CM, Chen Y, Sullivan ML, Kochanek PM and Clark RS Autophagy in acute brain injury: Feast, famine, or folly? Neurobiol Dis 43: 52-59, 2011.

22. Chen HC, Fong TH, Lee AW and Chiu WT: Autophagy is activated in injured neurons and inhibited by methylprednisolone after experimental spinal cord injury. Spine (Phila Pa 1976) 37: 470-475, 2012.

23. Kang R,Zeh HJ, Lotze MT and Tang D: The Beclin 1 network regulates autophagy and apoptosis. Cell Death Differ 18: 571-580, 2011.

24. Tanabe F, Yone K, Kawabata N, Sakakima H, Matsuda F, Ishidou Y, Maeda S, Abematsu M, Komiya S and Setoguchi T: Accumulation of p62 in degenerated spinal cord under chronic mechanical compression: Functional analysis of p62 and autophagy in hypoxic neuronal cells. Autophagy 7: 1462-1471, 2011.
25. Gurusamy N and Das DK: Detection of cell death by autophagy. Methods Mol Biol 559: 95-103, 2009.

26. Zhang Z, Zhang L, Yepes M, Jiang Q, Li Q, Arniego P, Coleman TA, Lawrence DA and Chopp M: Adjuvant treatment with neuroserpin increases the therapeutic window for tissue-type plasminogen activator administration in a rat model of embolic stroke. Circulation 106: 740-745, 2002.

27. Lv YC, Shi DY, Jiang XX, Feng ZZ, Jiang C and Chen ZX: Repair effects of neuroserpin (NSP) on neural function after acute spinal cord injury (SCI) in rats. Fudan Univ J Med Sci 41: 216-221, 2014 (In Chinese).

This work is licensed under a Creative Commons Attribution-NonCommercial-NoDerivatives 4.0 International (CC BY-NC-ND 4.0) License. 